\title{
Hemolytic Crisis after AB0-Nonidentical Solid Transplantation: Case Report
}

\author{
Irina Pashkova, Irina Gilevich and Vladimir Porhanov \\ Krasnodar Region Hospital \#1, Krasnodar, Russia
}

Correspondence should be addressed to: Irina Gilevich; giliv@list.ru

Received Date: 22 April 2014; Accepted Date: 5 June 2014; Published Date: 26 June 2014

Academic Editor: Burak Uz

Copyright (C) 2014 Irina Pashkova, Irina Gilevich and Vladimir Porhanov. Distributed under Creative Commons CC-BY 3.0

\begin{abstract}
Passenger lymphocyte syndrome develops after non-identical solid transplantations and may cause acute hemolytic anemia. A standard treatment for such patients is not known. We have reported 2 cases of hemolytic anemia developed during the early postoperative period after liver transplantation and our therapeutic approach.

Both patients showed AB Rh (D) positive blood type. The donors had B Rh (D) positive. Pretransplant HLA screening showed lack of antibodies. The first patient developed severe anemia on 16 posttransplantation day. The second patient was noticed with decreased hemoglobin and the increased of the liver enzymes on 5 day after transplantation. The liver biopsy of the second patient showed immunological damage features. In both cases anti-A1 antibodies were determined by indirect antiglobulin test (IAT) applying elution techniques, heat autocontrol was also positive. In all patients the direct antiglobulin test (DAT) was positive. The first patient was in stable conditions and did not demand any specific therapy. In the second case the pulse therapy with methylprednisolone (MP), and the plasmapheresis was initiated. Both patients were transfused with 0 RBC washed concentrates. The DAT became negative by 40 day after surgery. The patients were discharged in stable conditions after performed treatment.

Since we suppose the possible development of hemolytic crisis after ABO non-identical transplantation in future, we have included the immunohematological assays such as monitoring DAT along with HLA antibody screening in post-transplant period. In our opinion, the results of DAT and the antibodies specificity in eluate should be considered when transfusion of RBC is indicated.
\end{abstract}

Keywords: Passenger lymphocyte syndrome, hemolytic anemia, transplantation.

\section{Introduction}

One of the most major problems of transplantology remains the acute shortage of donor organs. Those are the reasons why in some cases, for example, when the recipients urgently need the donor organ transplant, it is possible to use compatible but not identical $\mathrm{ABO}$ organs. ABOcompatible organ transplantation may lead to expansion of the donor pool. However, it can increase the risk of complication

Cite this Article as: Irina Pashkova, Irina Gilevich and Vladimir Porhanov (2014), "Hemolytic Crisis after AB0-Nonidentical Solid Transplantation: Case Report," Research in Immunology: An International Journal, Vol. 2014 (2014), Article ID 999630, DOI: 10.5171/2014.999630 
development associated with immune ABO antibodies. One of them is acute or chronic hemolytic anemia. Immune-mediated hemolytic anemia occurs occasionally after transplantation, particularly after bone marrow or peripheral blood progenitor cell transplantation. Less frequently, it has been reported in solid organ transplantation (Sokol, R. J., et. al., 2002; Bakr, M. A., et. al., 2009).

Immune-mediated hemolysis after solid transplantation can be resulted in the passenger lymphocyte syndrome (PLS) (Bakr, M.A., et. al., 2009). The development of PLS is associated with surviving lymphocytes in the transplanted organ with the capability of reacting to some antigen in the recipient cells.

Our report reviewed two cases of hemolytic anemia after liver transplantation due to the PLS. The aim of our study was to analyse these cases and to determine in the future the order of the immunohematological assays for control the development of hemolytic complications and proper initiation the appropriate therapy.

\section{Materials and Methods}

In this report we studied the data of 386 solid transplantations. Among them only 45 transplantation were non-identical but compatible.

Before transplantation we performed immunological assays in all patients: HLAphenotyping and screening for the detection HLA-antibody, cross-match. The group blood ABO and Rhesus as alloantibodies were also observed. Serum samples of patients undergoing solid transplantation were collected during hospitalization and at regular outpatient visits. All positive samples of DAT were further examined by the gel agglutination and elution tests. The tests were performed according to the manufacturer's instructions. In perioperative period patients received immunosuppressive drugs according to standard therapy solid transplantations.

\section{HLA-Typing}

The detection of antigens of HLA-A, B - loci class I was performed by using electrophoresis method with reagents Dynal Resolusion (Invitrogen, USA);

We performed genotyping HLA II class with reagents HLA-DRB1 (DNA-Technology, Russia) using PCR-SSP method. In the case of ambiguous results or uncertainty, additional testing by PCR-SSOP Luminex (One Lambda, Inc., Canoga Park, CA. USA) was implemented for genotyping HLA -A, B, - DR.

\section{Screening and Determination of Antibody Specificity for HLA Class I and Class II}

The screening and identification of HLAantibody against class I and II were accomplished by ELISA method with reagents Quikscreen, B-Screen, Quik-ID Class I, II (GTI Diagnostics, USA).

Invitrogen HLA-FLT Trays (Invitrogen, USA) were used for the detection and identification antibody of Class I by the method of complement-dependent cytotoxicity.

For screening HLA-antibody we additionally performed PCR-SSOP Luminex (One Lambda, Inc., Canoga Park, CA. USA). Antibody specificity was analyzed manually using baseline (median fluorescence intensity values) and Ratio Scoring scales.

All tests were performed according to the vendor instructions.

\section{Donor-Specific Crossmatch (XM) Assay}

For donor-specific crossmatch two methods were included: complementdependent cytotoxicity XM and PCR-SSOP Luminex method. 


\section{Immunohematological Assays}

Immunohematology standard methods were used for serological phenotyping, antibodies identification and elution. For detecting the blood group of the recipient DG Gel ABO/Rh AB-AB-D-D'-ctl-A1-B crossgel method cards (Grifols, Spain) were used.

DAT and indirect antiglobulin test (IAT) were performed by gel cards (DG Gel Coombs, Grifols, Spain). The gel cards for DAT contained microcolumns filled with antiglobulin-monospecific serum against IgG, IgA, IgM, C3c, and C3d (Grifols, Spain). The antibody screen method included a $37^{\circ} \mathrm{C}$ incubation phase by an IAT using monospecific reagent (anti-IgG).

\section{Immunosuppression Protocol}

During the operation the patients received induction therapy: antagonist of Interleikin-2 (basiliximab) and steroids. All recipients were treated with a standard immunosuppression regimen, including calcineurin-inhibitor (cyclosporine (CsA) or tacrolimus), antiproliferative drug (azathioprine, mycophenolate mofetil (MMF) or sirolimus).

\section{Results}

There were 45 patients undergoing compatible but non-identical solid organ transplantation. We identified all ABOtransplantations in groups according to the organs presented in Table 1.

Table 1: The Characteristics of AB0 Non-Identical Transplantation

\begin{tabular}{|c|c|c|c|}
\hline $\begin{array}{l}\text { Patient blood } \\
\text { group }\end{array}$ & $\begin{array}{l}\text { Donor blood } \\
\text { group }\end{array}$ & $\mathbf{N}$ & $\%$ \\
\hline A & 0 & 12 & 26,7 \\
\hline B & 0 & 12 & 26,7 \\
\hline AB & 0 & 4 & 8,9 \\
\hline AB & A & 9 & 20 \\
\hline AB & B & 8 & 17,7 \\
\hline Total & & 45 & $100 \%$ \\
\hline
\end{tabular}

There were 27 males and 18 females and the age of recipient was $40 \pm 20$ years. Of those, 21 patients received the heart transplantation, 6 recipients - the liver, 18 recipients - the kidney. The antibodyinduced hemolysis induced by PLS, most frequently occurred in solid organ transplants, as heart-lung and kidney transplants, and rare in liver transplant (Petz, L. D., 2005; Ramsey, G., 1991).
We noted in our study, that in spite of the number of non-identical transplantation of hearts and kidneys were more than the liver transplantation, but there were no cases of hemolysis. We described the cases of hemolytic reaction in patients with the liver transplantation. Clinical characteristics of the studied patients are presented in Table 2. 
Table 2: Clinical Characteristics of the Studied Patients

\begin{tabular}{|c|c|c|}
\hline Characteristics of patients & Case 1: acute hemolysis & Case 2: acute hemolysis \\
\hline Patients age/ sex & $60 /$ female & $49 /$ male \\
\hline Donor blood group & $\mathrm{B}$ & $\mathrm{B}$ \\
\hline Recipient blood group & $\mathrm{AB}$ & $\mathrm{AB}$ \\
\hline HLA mismatching & 6 & 5 \\
\hline Direct antiglobulin test & ++ & +++ \\
\hline Antibody specificity & Anti-A & Anti-A \\
\hline Onset of hemolysis (day) & 16 & 5 \\
\hline Duration (day) & 24 & 20 \\
\hline $\begin{array}{l}\text { Histological evaluation of } \\
\text { liver biopsy at onset of } \\
\text { hemolysis }\end{array}$ & No & $\begin{array}{l}\text { The positive fixation of IgM, } \\
\text { IgA, C4d on endothelium of } \\
\text { hepatic sinusoid }\end{array}$ \\
\hline $\begin{array}{l}\text { The level of CsA at onset of } \\
\text { hemolysis, }(\mathrm{ng} / \mathrm{mL})\end{array}$ & 214 & 194,2 \\
\hline $\begin{array}{l}\text { Immunosuppression } \\
\text { before onset of hemolysis }\end{array}$ & $\mathrm{CsA}+\mathrm{MMF}+\mathrm{MP}$ & $\mathrm{CsA}+\mathrm{MMF}+\mathrm{MP}$ \\
\hline Treatment & Transfusion & $\begin{array}{l}\text { Transfusion; } \\
\text { plasmapheresis }\end{array}$ \\
\hline Outcome & Alive & Alive \\
\hline
\end{tabular}

CsA - cyclosporine, MMF - mycophenolate mofetil, MP - methilprednisolone.

\section{Patient 1}

A 60-year-old woman with the end-stage liver cirrhosis secondary to viral B etiology received a cadaveric liver transplant. Her pretransplant blood type was $\mathrm{AB}$ Rh (D) positive. IAT was negative. Her HLA phenotype was A 02, B 45 (12), B 27, DR 15 , DR 16. The pretransplant antibodies were not detected. Cytomegalovirus (CMV) serology before transplantation was IgG positive, IgM - negative; HBV and HCV serology were negative; PCR HCV was negative.

The donor blood group was $\mathrm{B}$ Rh (D) positive. HLA phenotype was A 03, A 26 (10), B 07, B 62 (15), DR 01, DR 17. CMV serology was IgG-positive, IgM-negative, PCR HCV, HCV and HBV serology were negative. Donor-specific XM was negative.

Intraoperative blood loss was $800 \mathrm{ml}$. She was transfused fresh frozen plasma (FFP) $\mathrm{AB}$ group $1800 \mathrm{ml}$ due to coagulation abnormalities as a result of liver disease, and autologous red blood was reinfused $350 \mathrm{ml}$.

During the operation the patient was treated with standard induction immunosuppression: basiliximab and methylprednisolone (MP).

In the postoperative period the patient received maintenance immunosuppressive therapy for the liver transplant, which consisted of MP, CsA and MMF. During the early postoperative period the patient had an anemia (hemoglobin - $83 \mathrm{~g} / \mathrm{L}$, the number of RBC - 2, $87 \times 10^{12} / \mathrm{L}$, hematocrit - 23), therefore she received 1 unit of RBC. After transfusion hemoglobin was in stable range, the liver enzymes decreased gradually over the past two weeks. On 16 day after transplantation the patient felt weakness, giddiness, and tachycardia without explanation. There were no signs of bleeding, and no signs of viral and/or bacterial infections. The liver enzymes (ALT and AST) were in normal range; total bilirubin was above normal range - 45, 7 $\mu \mathrm{mol} / \mathrm{L}$ (normal, $10-25 \mu \mathrm{mol} / \mathrm{L}$ ), direct bilirubin was 20, $7 \mu \mathrm{mol} / \mathrm{L}, \mathrm{LDH}-735,4$ U/L (normal, 208, 0 - 378, 0 U/L). The laboratory analyses revealed severe anemia with hemoglobin - $62 \mathrm{~g} / \mathrm{L}$, the number of RBC - 1, $5 \times 10^{12} / \mathrm{L}$, hematocrit 17,0 , reticulocytes were $5 \%$ (normal, $0-3$ $\%$ ); her platelet count was (PLT) $364 \mathrm{x}$ $10^{\%} / \mathrm{L}$; leucocytes were 15, $92 \times 10^{9} / \mathrm{L}$. 
Serum free hemoglobin was 0, $29 \mathrm{~g} / \mathrm{L}$ (normal range $\leq 0,4 \mathrm{~g} / \mathrm{L}$ ).

The crossmatch with $\mathrm{AB}$ group erythrocyte was positive $(2+)$ in the IAT and heat autocontrol was also positive $(2+)$. HLA antibody was not detected.

Additional investigations confirmed the presence of hemolysis, including a positive DAT. We determined fixed alloantibodies class IgG and C3d on the recipient's erythrocytes. Using elution techniques we identified anti-A1 antibody by IAT. Anti-A1 antibodies were detected in the patient's serum with agglutination scored $2+$, but they were not reactive to a titer 2 by IAT. As the titer of an antibody was low, and the patient was in a stable condition, we decided not to apply the plasmapheresis; the dose of immunosuppressive drug was not changed.

The patient was transfused with 3 units 0 of RBC washed concentrates, without complications. The crossmatch with 0 group erythrocyte was negative in the IAT and in elute IAT. After the transfusion blood parameters increased: hemoglobin $105 \mathrm{~g} / \mathrm{L}$, the number of RBC 3, $1 \times 10^{12} / \mathrm{L}$, hematocrit 29, 4 .

Further DAT was regularly investigated and it became negative to 40 day after surgery. She was discharged in stable condition.

\section{Patient 2}

A 49-year-old man with the terminal stage liver cirrhosis secondary to viral $\mathrm{C}$ etiology underwent a cadaveric liver transplantation. He had blood type $\mathrm{AB} \mathrm{Rh}$ (D) positive. IAT was negative. His HLA phenotype was A 23 (9), A 32 (19), B 35, B 41 , DR 13. The pretransplant antibodies were not detected. CMV serology before transplantation was IgG positive, IgMnegative; HBV serology was negative, antibodies to HCV were positive; PCR HCV was negative.

The donor blood group was $B$ Rh (D) positive. HLA phenotype was A 02, A 32 (19), B 13, B 27, DR 07, DR 16. CMV serology was IgG-positive, IgM-negative; PCR HCV, HCV and HBV serology were negative. Donor-specific XM was negative.

Intraoperative blood loss was $450 \mathrm{ml}$. Cell salvage was $150 \mathrm{ml}$. He was transfused FFP $1200 \mathrm{ml}$ due to coagulation abnormalities as a result of liver disease.

In the postoperative period the patient received maintenance immunosuppression, which consisted of MP, CsA and MMF.

In the early postoperative period no complications were observed, but the hemoglobin level decreased slowly. The liver enzymes began to decrease, but bilirubin was progressively increased. On day 5 after transplantation we noticed the decreased hemoglobin and increased liver enzymes. The patient examination showed no evidence of active bleeding. The laboratory analyses revealed the following: $\mathrm{Hb}-95 \mathrm{~g} / \mathrm{L}, \mathrm{RBC}-2,79 \times 10^{12} / \mathrm{L}, \mathrm{Ht}-26$, 3 , reticulocytes - 9\%, ALT - $281 \mu \mathrm{mol} / \mathrm{L}$, AST - $83 \mu \mathrm{mol} / \mathrm{L}$, and serum bilirubin $148 \mu \mathrm{mol} / \mathrm{L}$, direct bilirubin was 120 $\mu \mathrm{mol} / \mathrm{L}, \mathrm{LDH}$ - $938 \mathrm{U} / \mathrm{L}$. Serum free hemoglobin was $0,6 \mathrm{~g} / \mathrm{L}$.

On the same day the liver biopsy specimens were evaluated. Histological and immunofluorescence findings were similar to antibody-mediated rejection: IgG fixation was negative, IgM fixation and IgA fixation (on endothelium of hepatic sinusoid) were positive, $\mathrm{C} 1 \mathrm{q}$ fixation and C3 fixation were negative, but C4d fixation was positive (on endothelium of hepatic sinusoid). We found the features of immunological damages: the fixation of humoral factors on endothelium of hepatic sinusoid, but this mismatched to humoral crisis (the absence of the fixation in the intima of arterioles and venules), besides HLA - antibodies were not detected in serum.

Keeping in mind a possibility of hemolysis, immunohematological investigations were done, DAT was positive. The agglutination was scored as $3+$. We determined fixed alloantibodies class IgG and C3d on the recipient's erythrocytes by DAT. We identified anti-A1 antibodies by IAT used 
elution techniques, heat autocontrol was also positive.

On the basis of the clinical symptoms and immunological data of the laboratory investigations, the immunofluorescent findings were considered as the results of PLS.

The pulse therapy with MP (day 1, $500 \mathrm{mg}$; day 2, $250 \mathrm{mg}$; day 3, $125 \mathrm{mg}$ ), and the plasmapheresis were initiated. The volume of plasma exchange was $2400 \mathrm{ml}$. We have also raised the dosage of CsA to $350 \mathrm{mg}$.

But on day 9 his $\mathrm{Hb}$ level reached $65 \mathrm{~g} / \mathrm{L}$, which also confirmed our provisional diagnosis. Anemia was treated by 0 blood washed RBC. On day 20 laboratory markers of hemolysis were negative, $\mathrm{Hb}$ level increased to $93 \mathrm{~g} / \mathrm{L}$, bilirubin decreased to $57 \mu \mathrm{mol} / \mathrm{L}$. On day 40 , the DAT result was negative, $\mathrm{Hb}$ was $105 \mathrm{~g} / \mathrm{L}$, bilirubin was $19,5 \mu \mathrm{mol} / \mathrm{L}$. The patient was discharged with stable hemoglobin of 100 $\mathrm{g} / \mathrm{L}$ and immunosuppression, consisting of CsA and MMF.

\section{Discussion}

In this paper we would like to share the experience of the management of patients with hemolytic crisis. Since it was the first cases of hemolytic crisis in our observation, it attracted a special interest. We treated patient according to the surgeon indication with transfusion therapy. The selection of RBC was complex, and further investigation revealed the presence of hemolytic anemia in patients. Though, the anemia in perioperative period could be due to different reasons as the results of bleeding, drug therapy, the inflammatory process, the graft rejection. Biochemical markers such as increased indirect bilirubin, LDH in patients with hepatic pathology can be observed in the absence of hemolysis. We experienced some difficulties while starting diagnosis of acute hemolytic crisis.

Immune-mediated hemolysis in adult patients after solid organ transplantation has been reported in many papers (Sokol, R.J., et. al., 2002; Debska-Slizien A., et. al., 2003; Ainsworth, C.D., et. al., 2009). The hemolytic anemia may be related to antierythrocyte antibodies and generally it is induced by the PLS. The PLS syndrome developed as a result of the presence of donor passenger lymphocytes retained in transplanted organ. B-lymphocytes remain viable and can be capable of reacting to foreign antigens and produce adequate amounts of antibodies, especially blood group antigens (Bakr, M. A., et. al., 2009; Borka, P., et. al., 2001; Tiplady, C. W., et. al., 2001; Sternberg, A. J., et. al., 2000). Blymphocytes passengers interact with erythrocytes antigens of recipient and begin to produce antibodies specific AB0. The immune-mediated hemolysis usually has an abrupt onset and takes place in the early postoperative period of 120 days (Rougier J. P. et. al., 1994, Debska-Slizien A. and et.al., 2003). The clinical severity varies from self-limited to serious anemia causing to special therapy and transfusion management.

Patients of $A, B$ or $A B$ blood groups may receive organs from $\mathrm{ABO}$-compatible, although non-identical donors (mostly from 0 blood group donors). PLS may also happen, when patients of the $A B$ blood group receive an organ from a donor of the A or B blood groups. (Bakr, M. A., et. al., 2009, Petz, L. D., 2005, Ramsey, G., 1991). We found in the literature described with cases of hemolytic anemia mostly often developed after heart and lung transplantation (Chandra A. et. al., 2012, Ainsworth C. D., et. al. 2009, Ramsey, G., 1991).

Ramsey reported the frequency of hemolysis after liver transplantation. In a retrospective study including almost 1000 liver transplantations, in 782 patients were found antibodies against ABO antigens, in 22 of $60(36.7 \%)$ patients submitted to ABO compatible non-identical transplantation and showed that 13 of 22 (59.1\%) patients with antibodies had clinical hemolysis. (Ramsey G. and et. al., 1989, Chandra A. and et. al., 2012).

We analyzed 45 cases of ABO non-identical compatible solid organ transplantation, and anti-AB0 antibodies were observed in 6 patients. A hemolytic crisis, which 
required treatment, developed in only 2 patients with liver transplantation.

We identified the first case of hemolytic crisis development in 16 days after the transplantation. In most reported cases, the alloantibodies usually developed between 5 and 15 days after transplantation (Bakr, M. A., et. al., 2009, Sokol, R. J., 2002, Petz, L.D. 2005).

However, the clinical picture observed in this patient did not show the specific features of hemolytic crisis. Besides signs of anemia such as the low hemoglobin, we noted no increase of markers of pigment metabolism, or serum free hemoglobin. Only further observation, the lack of therapeutic effect, including transfusion, and exclusion of other causes reduction of hemoglobin, led us to think about the development of hemolysis and conducting special analyses.

The patient stable condition did not require the plasma exchange and pulse therapy, as well as correction of the immunosuppression therapy. After the transfusion RBC the patient noted health improvement. Later the level of hemoglobin continued to be stable above $90 \mathrm{~g} / \mathrm{L}$.

The second case occurred on day 5 after transplantation. It was interesting, that the results of biopsy showed signs of immunological damage, while absent convincing signs of humoral rejection caused allo-HLA-antibody. The differential diagnosis was between acute humoral rejection and hemolytic crisis due PLS.

Hemolytic anemia based on the presence of severe anemia, hyperbilirubinemia, and increased serum free hemoglobin. We concluded that this hemolytic anemia developed as a result of anti-A1 antibody. The hemolysis was managed by the use of corticosteroids, the plasma exchange, and the increased dose of the immunosuppressive drugs (the dose of CsA). Such supportive care had a positive effect, the biomarkers were normalized, the hemoglobin increased, on 30 days further results of the biopsy showed no signs of immune damage.

Currently, the recommendations of management of patients with AB0 nonidentical transplantations are being described by the different centers, but there are no standards.

Therapeutic approach includes correction of immunosuppression, immune modulation (intravenous immunoglobulin, corticosteroid) and specifically targeting Blymphocytes using monoclonal antibodies such as rituximab. In most cases PLS can be treated only with transfusions. (Chandra A. and et. al., 2012, Panaro F. and et al., 2004).

Supposung the possible development of this complication after ABO non-identical transplantation in future we decided to define the order of postoperative management.

We have included the immunohematological assays such as monitoring DAT along with HLA antibody screening post-transplant period.

In our opinion, the results of DAT and the antibodies specificity in eluate should be considered when transfusion of $\mathrm{RBC}$ is necessary.

The correction of immunosuppressive therapy, the conduct of plasmapheresis will depend on the severity of hemolysis, and general clinical condition of the patient. We continue to collect data for further analysis as in the present paper we described too small number of observations.

\section{Abbreviations}

CMV: cytomegalovirus; CsA: cyclosporine; DAT: direct antiglobulin test; FFP: fresh frozen plasma; IAT: indirect antiglobulin test; MMF: mycophenolate mofetil; MP: methylprednisolone; PLS: passenger lymphocyte syndrome; PLT: platelet; RBC: red blood cells; XM: crossmatch. 


\section{References}

Ainsworth, C. D., Crowther, M. A., Treleaven, D., Evanovitch, D., Webert, K. E. \& Blajchman, M. A. (2009). "Severe Hemolytic Anemia Post-Renal Transplantation Produced by Donor Anti-D Passenger Lymphocytes: Case Report and Literature Review," Transfusion Medicine Reviews, 23 (2) 155-159.

Bakr, M. A., Abbas, T. M., Mustafa, A. \& Ghoneim, M. A. (2009). "Hemolytic Anemia after ABO Nonidentical Living Donor Kidney Transplantation," Clinical and Experimental Nephrology, 13 (2) 161-165.

Borka, P., Jakab, J., Rajczy, K., Remport, Á., Járay, J., Hoffer, I. \& Perner, F. (2001). "Temporary Donor-derived B-lymphocyte Microchimerism Leading to Hemolysis in Minor AB0-incompatible Renal Transplantation," Transplantation Proceedings, 33 (3) 2287-2289.

Chandra, A., Gupta, S. S., Sharma, R. K., Kaul, A., Bhadauria, D., Prasad, N., Gupta, A., Elhence, P., Sonkar, A. \& Chandra, H. (2012). "Passenger Lymphocyte Syndrome Exhibited in a Renal Transplant Recipient: A Case Report," Indian Journal of Transplantation, 6 (3) 97-99.

Debska-Slizien, A., Chamienia, A., Krol, E., Zdrojewski, Z., Pirski, I., Zadrożny, D., Sledzinski, Z. \& Rutkowski, B. (2003). "Hemolytic Anemia after Renal Transplantation: Analysis of Case Reports," Transplantation Proceedings, 35 (6) 22332237.

Panaro, F., De Christopher, P. J., Rondelli, D., Testa, G., Sankary, H., Popescu, M. \& Benedettil, E. (2004). "Severe Hemolytic Anemia Due to Passenger Lymphocytes after Living Related Bowel Transplant," Clinical Transplantation, 18 (3) 332-335.
Petz, L. D. (2005). "Immune Hemolysis Associated with Transplantation," Seminars in Hematology, 42 (3) 145-55.

Ramsey, G. (1991). "Red Cell Antibodies Arising from Solid Organ Transplants," Transfusion, 31 (1) 76-86.

Ramsey, G., Cornell, F. W., Hahn, L. F., Larson, P., Issitt, L. B. \& Starzl, T. E. (1989). "Red Cell Antibody Problems in 1000 Liver Transplants," Transfusion, 29 (5) 396-400.

Rougier, J. P., Viron, B., Ronco, P., Khayat, R., Micheal, C. \& Mignon, F. (1994). "Autoimmune Haemolytic Anaemia after ABO-Match, ABDR Full Match Kidney Transplantation," Nephrology Dialysis Transplantation, 9 (6) 693-697.

Sokol, R. J., Stamps, R., Booker, D. J., Scott, F. M., Laidlaw, S. T., Vandenberghe, E. A. \& Barker, H. F. (2002). "Posttransplant Immune-Mediated Hemolysis," Transfusion, 42 (2) 198-204.

Sternberg, A. J., Lee, G., Croxton, T., Mundy, N., Morris, P., Littlewood, T. J. \& Murphy, M. F. (2000). "Severe Haemolysis after an ABO Unmatched Kidney Transplant - A Non Secretor Transplanted from a Donor with High Anti-A Titer," Transfusion Medicine, 10 (1) 87-89.

Tiplady, C. W., Fitzgerald, J. M., Jackson, G. H., Conn, J. S. \& Proctor, S. J. (2001). "Massive Haemolysis in a Group a Recipient of a Group 0 Peripheral Blood Stem Cell Allogeneic Transplant," Transfusion Medicine, 11 (6) 455-458. 